\title{
Predictors of Neuropsychiatric Adverse Events with Smoking Cessation Medications in the Randomized Controlled EAGLES Trial
}

\author{
Robert M. Anthenelli, MD' ${ }^{7}$, Michael Gaffney, PhD², Neal L. Benowitz, MD ${ }^{3}$, Robert West, PhD', \\ Thomas McRae, $M D^{2}$, Cristina Russ, $M D^{2}$, David Lawrence, $P h D^{2}$, Lisa St. Aubin, DVM², \\ Alok Krishen, $M S^{5}$, and A. Eden Evins, $M D^{6}$
}

'Department of Psychiatry, University of California, San Diego, La Jolla, CA, USA; ${ }^{2}$ Pfizer, New York, NY, USA; ${ }^{3}$ Department of Medicine, University of California, San Francisco, CA, USA; “ University College, London, UK; ${ }^{5}$ PAREXEL International on behalf of GlaxoSmithKline, Research Triangle Park, NC, USA; ${ }^{M}$ Massachusetts General Hospital and Harvard Medical School, Boston, MA, USA.

BACKGROUND: Pre-treatment factors that increase smokers' risk of experiencing neuropsychiatric adverse events (NPSAEs) when quitting smoking are unknown.

OBJECTIVE: To identify baseline smoker characteristics beyond the history of mental illness that predict which participants were more likely to experience moderate to severe NPSAEs in EAGLES.

DESIGN: A prospective correlational cohort study in the context of a multinational, multicenter, double-blind, randomized trial.

PARTICIPANTS: Smokers without ( $N=3984$; NPC)/with $(N=4050 ; \mathrm{PC})$ histories of, or current clinically stable, psychiatric disorders including $\operatorname{mood}(N=2882 ; 71 \%)$, anxiety $(N=782 ; 19 \%)$, and psychotic $(N=386 ; 10 \%)$ disorders.

INTERVENTIONS: Bupropion, 150 mg twice daily, or varenicline, $1 \mathrm{mg}$ twice daily, versus active control (nicotine patch, $21 \mathrm{mg} /$ day with taper) and placebo for 12 weeks with 12-week non-treatment follow-up.

MAIN MEASURES: Primary safety outcome was the incidence of a composite measure of moderate/severe NPSAEs. Associations among baseline demographic/ clinical characteristics and the primary safety endpoint were analyzed post hoc via generalized linear regression. KEY RESULTS: The incidence of moderate to severe NPSAEs was higher among smokers in the PC (238/ $4050 ; 5.9 \%)$ than in the NPC $(84 / 3984 ; 2.1 \%)$. Three baseline characteristics predicted increased risk for experiencing clinically significant NPSAEs when quitting regardless of carrying a psychiatric diagnosis: current symptoms of anxiety (for every 4-unit increase in HADS anxiety score, the absolute risk of occurrence of the NPSAE endpoint increased by $1 \%$ in both PC and NPC); prior history of suicidal ideation and/or behavior (PC, $4.4 \%$ increase; $P=0.001 ;$ NPC, $4.1 \%$ increase; $P=0.02$ ), and being of White race (versus Black: $\mathrm{PC}, 2.9 \% \pm 0.9$ [SE] increase; $P=0.002$; and NPC, $3.4 \% \pm 0.8$ [SE] increase; $P=0.001)$. Among smokers with psychiatric disorders, younger age, female sex, history of substance use disorders, and proxy measures of nicotine dependence or psychiatric illness severity also predicted greater risk. There were no significant interactions between these

Received March 12, 2018

Revised September 5, 2018

Accepted January 11, 2019

Published online March 7, 2019 characteristics and treatment. Smokers with unstable psychiatric disorders or with current, active substance abuse were excluded from the study.

CONCLUSIONS: Irrespective of cessation pharmacotherapy use, smokers attempting to quit were more likely to experience moderate to severe NPSAEs if they reported current anxiety or prior suicidal ideation at baseline and were White. In smokers with a psychiatric history, female sex, younger age, and greater severity of nicotine dependence were also predictive.

TRIAL REGISTRATION: ClinicalTrials.gov Identifier: NCT01456936

KEY WORDS: neuropsychiatric adverse event; smoking cessation medication; varenicline; predictors.

J Gen Intern Med 34(6):862-70

DOI: $10.1007 / \mathrm{s} 11606-019-04858-2$

(c) Society of General Internal Medicine 2019

\section{INTRODUCTION}

The large, multinational, Evaluating Adverse Events in a Global Smoking Cessation Study (EAGLES; NCT01456936) ${ }^{1}$ found that $\sim 4 \%$ of smokers experienced a clinically significant neuropsychiatric adverse event (NPSAE) during a quit attempt regardless of the medication to which they were assigned: transdermal nicotine patch (nicotine replacement therapy, NRT), bupropion, varenicline, or placebo. NPSAE incidence across active treatments was not significantly different from placebo. Incidence varied significantly by cohort: $\sim 2 \%$ of smokers without a history of a psychiatric disorder (non-psychiatric cohort, NPC) reported clinically significant NPSAEs, compared with $\sim 6 \%$ of smokers with current stable or past psychiatric disorders (psychiatric cohort, PC). However, little is known about patient-level characteristics or disease-related factors that predict which smokers are more likely to experience clinically significant NPSAEs when trying to quit. Identifying such pre-existing factors might help clinicians anticipate which smokers are at heightened risk and modify treatment accordingly.

Studies evaluating predictors of AEs with smoking cessation pharmacotherapies are sparse. The largest study in transdermal nicotine patch users found that successfully quitting 
smoking and female sex predicted sleep disturbances, and that application site reactions were associated with other skin conditions, younger age, female sex, and other demographic characteristics. $^{2}$ Given that some neuropsychiatric side effects from pharmacotherapy might overlap with symptoms of nicotine withdrawal, it is relevant that several studies have evaluated predictors of withdrawal and found that changes in mood states such as anhedonia, ${ }^{3}$ negative affect ${ }^{4}$ and anxiety, ${ }^{5,6}$ and severity of nicotine dependence ${ }^{7}$ are associated with nicotine withdrawal symptom expression. However, no study has examined NPSAEs among smokers in depth, and none have compared the relative risk across all three frontline medications and placebo in smokers with and without psychiatric conditions until now.

The purpose of this secondary cohort analysis was to go beyond the pre-specified analyses described in our prior work to examine baseline characteristics associated with the trial's primary composite safety endpoint to determine potential predictors of clinically significant NPSAEs in participants from EAGLES. In models that considered whether the treatments themselves were associated with such adverse outcomes, we explored whether demographic and clinical characteristics of the smokers, smoking-related variables, and mental healthrelated characteristics were associated with these adverse effects.

\section{METHODS}

\section{Design Overview}

EAGLES was a phase 4, multinational, multicenter, randomized, double-blind, triple-dummy, active- (NRT), and placebocontrolled trial of varenicline and bupropion for 12 weeks with 12-week non-treatment follow-up conducted between November 2011 and January 2015, at 140 centers in 16 countries. ${ }^{1}$ Details of the methods were described previously. ${ }^{1}$

The institutional review boards at participating institutions approved consent forms and the study procedures (the study protocol is available at http://www.thelancet.com/journals/lancet/article/PIIS0140-6736(16)30272-0/abstract). The study adhered to the Declaration of Helsinki ${ }^{8}$ and the International Conference on Harmonization Good Clinical Practice Guidelines. ${ }^{9}$ An independent Data Monitoring Committee reviewed safety data at pre-specified time points to ensure participant safety.

\section{Setting and Participants}

Eligible participants were men and women aged 18-75 years, who smoked an average of $\geq 10$ cigarettes per day during the prior year and during the month before the screening visit, had an exhaled carbon monoxide $(\mathrm{CO})>10 \mathrm{ppm}$ at screening, and who were motivated to stop smoking. All participants provided informed consent and were interviewed by trained mental health professionals using the valid and reliable ${ }^{10}$ Structured
Clinical Interviews for Diagnostic and Statistical Manual of Mental Disorders, Fourth Edition, Text Revision (DSM-IV$T R)^{11}$ for Axis I (SCID-I) ${ }^{12}$ and Axis II (SCID-II) ${ }^{13}$ disorders. Participants included in the NPC had no history of DSM-IV-TR Axis I disorders. Included in the PC were participants with a primary diagnosis of mood disorders (major depression, bipolar I or bipolar II disorder), anxiety disorders (panic disorder with or without agoraphobia, post-traumatic stress disorder, obsessive-compulsive disorder, social phobia, generalized anxiety disorder), psychotic disorders (schizophrenia, schizoaffective disorder), or borderline personality disorder meeting the $D S M-I V-T R^{11}$ criteria confirmed by the SCID-I and SCIDII. PC participants were clinically stable (i.e., no exacerbations of their condition in the prior 6 months; if on treatment, treatment was stable for at least 3 months, with no treatment change anticipated during the study) and not judged to be at high risk of self-injurious or suicidal behavior. Psychiatric comorbidities were allowed if not considered severe enough to compromise study participation. Participants with a primary diagnosis of borderline personality disorder were not included in the present analysis owing to the small size of the group not allowing proper statistical modeling. All other participants who were randomized and received at least one dose of study treatment were included in the analysis.

\section{Randomization and Interventions}

Participants were assigned to one of the two cohorts ( $\mathrm{PC}$ or NPC) on the basis of SCID results, with equal cohort sizes planned. They were then randomized in a 1:1:1:1 ratio using a computer-generated schedule to receive varenicline $1 \mathrm{mg}$ twice daily, bupropion $150 \mathrm{mg}$ twice daily, NRT $21 \mathrm{mg} /$ day with taper, or placebo for 12 weeks with a 12 -week nontreatment follow-up.

Treatment compliance was $\sim 80 \%$ across the four treatments. Participants were encouraged to complete all study visits even if treatment was discontinued.

\section{Outcomes and Follow-up}

The pre-specified primary outcome for EAGLES was also the outcome for this post hoc secondary cohort analysis and was defined as a composite measure of moderate to severe NPSAEs developed with input from the US Food and Drug Administration (FDA). The endpoint was operationalized by coding AEs reported by the investigators to preferred terms (PTs) in the Medical Dictionary for Regulatory Activities (MedDRA) and by further mapping those PTs in a predefined way to 16 neuropsychiatric (NPS) symptom categories included in the composite measure. In addition, NPSAEs (new events or increases in severity of ongoing symptoms) had to occur during treatment or within 30 days of treatment discontinuation and had to meet pre-established severity criteria to be included in the outcome. NPSAEs were required to be severe (significant interference with subject's usual daily functioning) for four of the 16 components (anxiety, depression, 
feeling abnormal, or hostility). The remaining 12 (agitation, aggression, delusions, hallucinations, homicidal ideation, mania, panic, paranoia, psychosis, suicidal ideation [SI], suicidal behavior [SB], or suicide, including suicidal depression) had to be rated as either moderate (some interference) or severe.

\section{NPSAEs and Psychiatric Rating Scales}

At baseline, cigarette dependence severity was assessed with the Fagerström Test for Cigarette Dependence (FTCD), ${ }^{14}$ and trait aggression was measured using the Buss-Perry Aggression Questionnaire (BPAQ). ${ }^{15}$ The Columbia-Suicide Severity Rating Scale (C-SSRS ${ }^{16}$ and the Hospital Anxiety and Depression Scale (HADS) ${ }^{17}$ a self-reported inventory that measures both anxiety (seven items) and depressive symptoms (seven items), were administered at baseline and all study visits to assess aspects of psychiatric symptom severity.

In addition, at all study visits, trained interviewers elicited the emergence of NPSAEs with open-ended questions, direct observation, and a semi-structured Neuropsychiatric Adverse Events Interview (NAEI). ${ }^{1,}{ }^{18}$ The NAEI is a 25 -item interview designed by Pfizer in consultation with psychiatric experts to specifically probe for psychiatric symptoms during the conduct of a clinical trial. A version of the instrument was first used in a randomized placebo-controlled trial of varenicline in smokers with major depressive disorder (NCT01078928) as described in Anthenelli et al. (2013). ${ }^{18}$ The instrument is designed to proactively solicit psychiatric symptoms and to supplement information that is volunteered by or observed of participants to determine whether a NPSAE may be present. Investigators then evaluated whether positive responses from these interviews, as well as on HADS and the C-SSRS, were NPSAEs. Additionally, proxy reports from subjects' family members or physicians were invited by asking participants to provide these collateral informants with cards that highlighted the NPSAEs of interest and directed them to contact the investigative study site if concerned about the study participants. This NPSAE assessment battery was designed a priori in consultation with the US FDA, codified in the study protocol, and utilized by trained personnel at each investigative site.

\section{Statistical Analysis}

The baseline covariates examined in this secondary cohort analysis were a combination of categorical and numerical measures: (1) demographic and clinical characteristics including age, sex, race (White, Black, Other), and body mass index (BMI); (2) smoking-related characteristics including severity of cigarette dependence (FTCD), age at onset of regular smoking, and prior use of smoking cessation study medications (no or any for each of the three medications); and (3) mental health-related measures including having any type of comorbid psychiatric diagnosis, a history of an alcohol or other substance use disorder, HADS anxiety subscale score, HADS depression subscale score, lifetime SI or SB as captured by C-SSRS, trait aggression as measured by the BPAQ, and use of psychotropic medications including sleeping aids. Within the PC, primary subcohort membership (i.e., primary mood, anxiety, or psychotic disorder) was also analyzed as a covariate.

Associations among covariates and the primary composite NPSAE outcome were analyzed with a sequential approach via generalized linear regression. For continuous covariates, a quadratic relationship was also investigated. As established in the main EAGLES outcome paper, ${ }^{1}$ a primary psychiatric diagnosis was a strong predictor of NPSAEs, and therefore the additional predictors of NPSAE were analyzed within the NPC and PC separately. Covariates and their by-treatment interactions deemed significant via a screening assessmentmodel controlling for treatment (NRT, bupropion, varenicline, and placebo) and region (US versus non-US) - were subsequently included in a stepwise regression analysis that determined those covariate terms contributing to the rate of NPSAE.

\section{RESULTS}

Figure 1 is a modified, abridged version of the CONSORT flow diagram presented in the primary manuscript. ${ }^{1}$ It differs in that 24 participants in the original PC sample with primary diagnoses of borderline personality disorder were excluded in this secondary analysis owing to the small sample size and modeling interference of this group; these individuals also did not report any primary endpoint NPSAEs. Overall, study discontinuation rates were similar across treatment groups during both the active treatment phase $(\mathrm{NPC}=14.6 \%$; $\mathrm{PC}=$ $15.4 \%)$ and the 12 -week non-treatment follow-up period $(\mathrm{NPC}=7.0 \% ; \mathrm{PC}=6.7 \%)$.

As partially reported previously, ${ }^{1} 84$ (2.1\%) of 3984 participants in the NPC, and 238 (5.9\%) of 4050 participants in the PC, reported moderate and severe NPSAEs.

\section{Baseline Characteristics}

Baseline demographic, smoking history, and psychiatric characteristics of the 8034 participants included in the present secondary analysis are illustrated in Table 1. As reported previously, baseline characteristics were similar across treatment groups but there were differences between the two cohorts. ${ }^{1}$ Those enrolled in the PC were more likely to be women, be recruited from sites within the USA, and have higher levels of cigarette dependence. More than one-third of those enrolled in the PC met the DSM-IV-TR criteria for a comorbid psychiatric disorder, and $23 \%$ had a history of an alcohol or other substance use disorder. As expected, baseline HADS anxiety and depression subscale scores were higher in PC smokers than smokers without psychiatric disorders, and the $\mathrm{PC}$ group reported prior histories of SI and/or SB that were more than sevenfold greater than NPC smokers. 


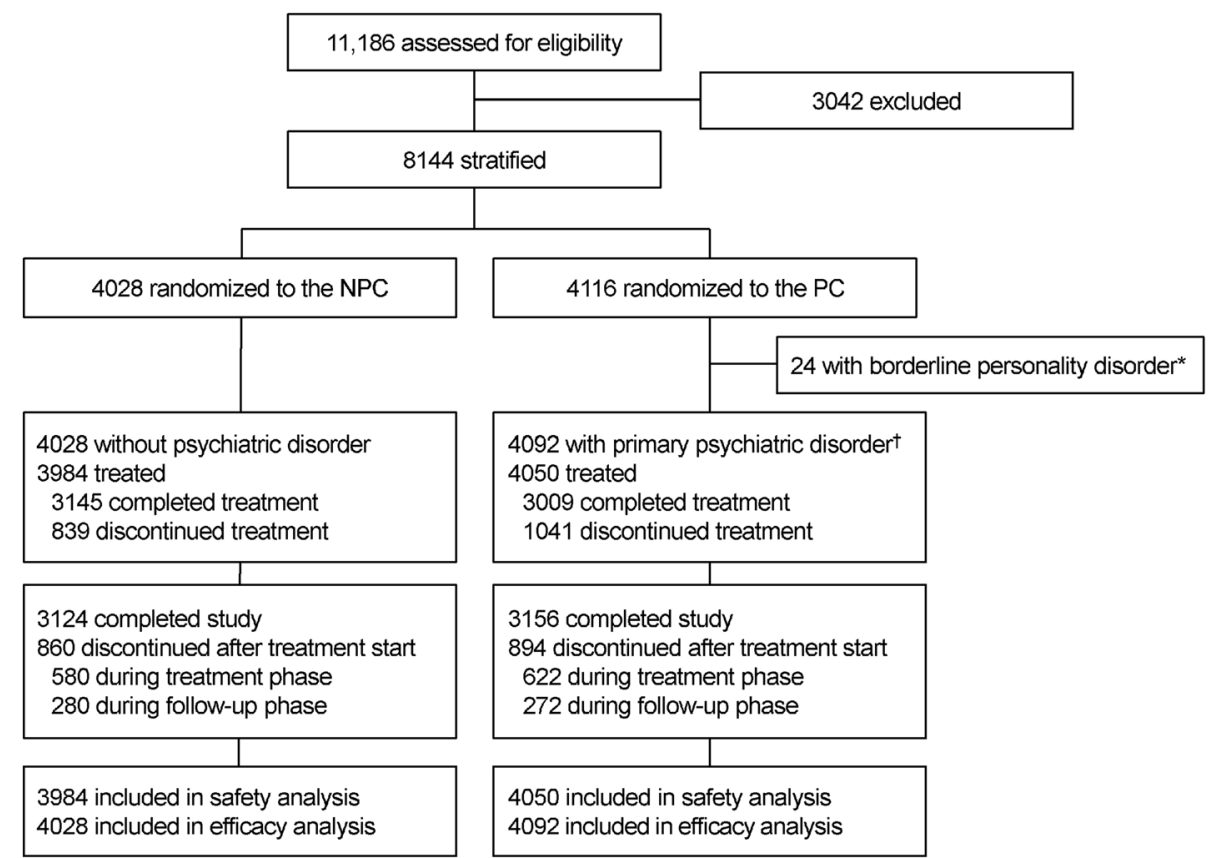

Fig. 1 Participant disposition (abridged CONSORT flow diagram). NPC non-psychiatric cohort, PC psychiatric cohort. The asterisk indicates exclusion from the present analysis because the small size of this group did not permit accurate modeling involving this disorder. The dagger indicates the primary diagnosis of mood, anxiety, or psychotic disorder.

\section{Predictors of NPSAEs in the PC}

Table 2 illustrates the regression results of the screening assessment of predictors in the PC. Twelve of the 17 predictor variables (italicized for emphasis) were significantly associated with an increased rate of occurrence of the primary NPSAE endpoint regardless of the participant's region of enrolment (US versus non-US). These included demographic/clinical characteristics (younger age, female sex, White versus Black race, and greater BMI), smoking-

Table 1 Baseline Demographic, Smoking, and Psychiatric Characteristics

\begin{tabular}{|c|c|c|}
\hline & NPC $(N=3984)$ & PC $(N=4050)$ \\
\hline Mean age (SD) (years) & $46.0(12.9)$ & $47.1(11.7)$ \\
\hline Male sex, $n(\%)$ & $2003(50.3)$ & $1542(38.1)$ \\
\hline \multicolumn{3}{|l|}{ Race, $n(\%)$} \\
\hline White & $3293(82.7)$ & $3268(80.7)$ \\
\hline Black & $504(12.7)$ & $640(15.8)$ \\
\hline Other & $187(4.7)$ & $141(3.5)$ \\
\hline Region* (USA), $n(\%)$ & $1875(47.1)$ & $2330(57.5)$ \\
\hline Mean BMI (SD) $\left(\mathrm{kg} / \mathrm{m}^{2}\right)$ & $27.6(6.1)$ & $28.6(6.6)$ \\
\hline Mean FTCD (SD) & $5.5(2.0)$ & $6.0(2.0)$ \\
\hline Mean age of starting smoking (SD) (years) & $17.9(5.3)$ & $18.5(6.5)$ \\
\hline Prior varenicline use, $n(\%)$ & $576(14.5)$ & $687(17.0)$ \\
\hline Prior bupropion ${ }^{\dagger}$ use, $n(\%)$ & $371(9.3)$ & 463 (11.4) \\
\hline Prior NRT use, $n(\%)$ & $994(24.9)$ & $1121(27.7)$ \\
\hline \multicolumn{3}{|l|}{ Primary psychiatric diagnosis, $n(\%)$} \\
\hline Mood disorder & N/A & $2882(71.2)$ \\
\hline Anxiety disorder & N/A & $782(19.3)$ \\
\hline Psychotic disorder & N/A & $386(9.5)$ \\
\hline Any comorbid psychiatric diagnosis, $n(\%)$ & $15(0.4)$ & $1461(36.1)$ \\
\hline Alcohol/substance use disorder history, $n(\%)$ & $8(0.2)$ & $932(23.0)$ \\
\hline Mean HADS anxiety total (SD) & $2.8(2.7)$ & $5.2(3.9)$ \\
\hline Mean HADS depression total (SD) & $1.5(2.1)$ & $3.2(3.3)$ \\
\hline Prior SI/SB (C-SSRS), $n(\%)$ & $194(4.9)$ & $1398(34.5)$ \\
\hline Mean aggression score (BPAQ) (SD) & $52.1(15.4)$ & $58.6(18.5)$ \\
\hline Baseline psychotropic medication, $n(\%)$ & $356(8.9)$ & $2261(55.8)$ \\
\hline
\end{tabular}

Abbreviations: BMI body mass index, BPAO Buss-Perry Aggression Questionnaire, C-SSRS Columbia-Suicide Severity Rating Scale, FTCD Fagerström Test for Cigarette Dependence, HADS Hospital Anxiety and Depression Scale, N/A not applicable, NPC non-psychiatric cohort, NRT nicotine replacement therapy (transdermal nicotine patch), $P C$ psychiatric cohort, SB suicidal behavior, SD standard deviation, SI suicidal ideation

*Non-US countries: Argentina, Australia, Brazil, Bulgaria, Canada, Chile, Denmark, Finland, Germany, Mexico, New Zealand, Russia, Slovakia, South Africa, and Spain

† Bupropion prior use for smoking cessation or other indications 
Table 2 Regression Results of Screening Assessment of Predictors of NPSAE (PC)

\begin{tabular}{|c|c|c|c|}
\hline Covariate & $\begin{array}{l}\text { Increase in rate of primary } \\
\text { NPSAE endpoint, \% (SE) }\end{array}$ & $\begin{array}{l}P \text { value for effect } \\
\text { of covariate }\end{array}$ & $\begin{array}{l}P \text { value for treatment- } \\
\text { by-covariate interac- } \\
\text { tion }\end{array}$ \\
\hline Age (1-year increase) & $\begin{array}{l}-0.1(0.0) \\
\text { Or } 1.0 \% \text { decrease per } 8.5 \\
\text { years increase in age }\end{array}$ & $<0.001$ & 0.46 \\
\hline Sex (male vs. female) & $-2.1(0.7)$ & 0.004 & 0.26 \\
\hline Race & & & 0.23 \\
\hline White vs. Black & $2.9(0.9)$ & 0.002 & - \\
\hline Other vs. Black & $2.7(2.3)$ & 0.25 & - \\
\hline BMI (1-unit increase) & $\begin{array}{l}0.1(0.1) \\
\text { Or } 1.0 \% \text { increase per 7.7-unit } \\
\text { increase in BMI }\end{array}$ & 0.03 & 0.27 \\
\hline FTCD* (1-unit increase) & $\begin{array}{l}0.4(0.2) \\
\text { Or } 1.0 \% \text { increase per 2.7-unit } \\
\text { increase in FTCD }\end{array}$ & 0.04 & 0.95 \\
\hline Age of starting smoking (1-year increase) & $-0.0^{\dagger}(0.1)$ & 0.34 & 0.13 \\
\hline Prior varenicline use (yes vs. no) & $1.6(1.1)$ & 0.15 & 0.35 \\
\hline Prior bupropion use (yes vs. no) & $5.1(1.5)$ & $<0.001$ & 0.30 \\
\hline Prior NRT use (yes vs. no) & $2.2(0.9)$ & 0.02 & 0.51 \\
\hline $\begin{array}{l}\text { Primary subcohort psychiatric diagnosis } \\
\text { (psychotic, anxiety, mood disorders) }\end{array}$ & -+ & 0.99 & 0.93 \\
\hline Any comorbid psychiatric diagnosis (yes vs. no) & $3.3(0.8)$ & $<0.001$ & 0.89 \\
\hline Alcohol/substance use disorder history (yes vs. no) & $3.8(1.0)$ & $<0.001$ & 0.96 \\
\hline HADS anxiety total (1-unit increase) & $\begin{array}{l}0.4(0.1) \\
\text { Or } 1.0 \% \text { increase per 2.7-unit } \\
\text { increase in HADS anxiety } \\
\text { total }\end{array}$ & $<0.001$ & 0.91 \\
\hline HADS depression total (1-unit increase) & $0.2(0.1)$ & 0.06 & 0.76 \\
\hline Prior SI/SB (C-SSRS) (yes vs. no) & $5.9(0.9)$ & $<0.001$ & 0.17 \\
\hline Aggression score (BPAQ) (1-unit increase) ${ }^{\S}$ & $0.0(0.0)$ & 0.63 & 0.66 \\
\hline Linear term & $0.2(0.1)$ & 0.04 & - \\
\hline Quadratic term & $-0.0(0.0)$ & 0.04 & - \\
\hline $\begin{array}{l}\text { Baseline psychotropic medication including sleeping aids (yes vs. } \\
\text { no) }\end{array}$ & $1.2(0.7)$ & 0.09 & 0.35 \\
\hline
\end{tabular}

Abbreviations: BMI body mass index, BPAQ Buss-Perry Aggression Questionnaire, C-SSRS Columbia-Suicide Severity Rating Scale, FTCD Fagerström Test for Cigarette Dependence, HADS Hospital Anxiety and Depression Scale, NPSAE neuropsychiatric adverse event, NRT nicotine replacement therapy (transdermal nicotine patch), PC psychiatric cohort, SB suicidal behavior, SE standard error, SI suicidal ideation

$*$ Categorization of FTCD into three categories was significant through its interaction with treatment $(P=0.04)$

+Denotes a negative value near zero

$¥$ The main effect of the psychiatric cohort diagnosis was not significant $(P=0.99)$, and estimates for pairwise comparisons have no predictive value and are not shown

${ }^{\xi}$ The aggression score was the only covariate with a non-linear relationship with NPSAE. A quadratic relationship between aggression score and NPSAE is significant; the quadratic fit indicates an increasing probability of NPSAE, which maximizes at a score of 58 and then turns downward A significant effect was found in the screening assessment for covariates in italics

Interactions among each baseline predictor and region (US versus non-US) were not statistically significant in the multivariate linear regression model and are not shown

related variables (higher level of cigarette dependence, prior bupropion use, and prior NRT use), and mental health-related characteristics (psychiatric comorbidity, past history of substance use disorder, elevated HADS anxiety score, lifetime history of SI/SB, and higher trait aggression). The table also shows the magnitude of the effect of the predictor and, if the predictor is a significant continuous variable (e.g., age), the change in units to yield a $1.0 \%$ increase in the chance of a clinically significant NPSAE. Primary psychiatric disorder diagnostic category (e.g., mood, anxiety, or psychotic disorder) as determined by the SCID-I was not significantly associated with NPSAEs.

The significant covariates specified above were entered in a stepwise final regression analysis to identify those predictor variables that independently contributed to the NPSAE rate. As illustrated in Table 3, seven predictors were retained in the model, including younger age, female sex, prior use of bupropion, prior NRT use, history of a substance use disorder, higher HADS anxiety score, and lifetime history of SI/SB.

\section{Predictors of NPSAEs in the NPC}

In contrast to the large number of variables found to be associated with an increased occurrence of clinically significant NPSAEs in the PC, Table 4 shows that in the NPC, only three factors were significantly associated with the primary safety endpoint, a result confirmed in the final regression model (see Table 5). Notably, these three factors - higher HADS anxiety scale score, endorsing a prior history of $\mathrm{SI} / \mathrm{SB}$, and White race-were consistently associated with increased NPSAE risk in both cohorts in the screening assessment (italicized in Table 2 and Table 4 for emphasis).

There were no significant treatment-by-covariate interactions in either cohort. 
Table 3 Results of Stepwise Regression Analysis of Predictors of NPSAE (PC)

\begin{tabular}{lll}
\hline \hline Covariate & $\begin{array}{l}\text { Increase in rate of primary } \\
\text { NPSAE endpoint, \% (SE) }\end{array}$ & $\begin{array}{c}\text { P value for effect } \\
\text { of covariate }\end{array}$ \\
\hline Age (1-year increase) & $-0.1(0.0)$ & 0.002 \\
& Or 1.0\% decrease per 10.8-year increase in age & 0.004 \\
Sex (male vs. female) & $-1.9(0.6)$ & 0.004 \\
Prior bupropion use (yes vs. no) & $4.0(1.4)$ & 0.01 \\
Prior NRT use (yes vs. no) & $1.9(0.8)$ & 0.01 \\
Alcohol/substance use disorder history (yes vs. no) & $2.4(0.1)$ & 0.003 \\
HADS anxiety total (1-unit increase) & $0.3(0.1)$ & \\
& Or 1.0\% increase per 3.9-unit increase in HADS & $<0.001$ \\
Prior SI/SB (C-SSRS) (yes vs. no) & anxiety total & \\
\hline
\end{tabular}

Abbreviations: C-SSRS Columbia-Suicide Severity Rating Scale, HADS Hospital Anxiety and Depression Scale, NPSAE neuropsychiatric adverse event, NRT nicotine replacement therapy (transdermal nicotine patch), PC psychiatric cohort, SB suicidal behavior, SE standard error, SI suicidal ideation

\section{DISCUSSION}

Regardless of a smoker's psychiatric history, being more anxious at treatment onset, having a history of prior SI or $\mathrm{SB}$, and being of White race were independently associated with an increased risk of experiencing a clinically significant NPSAE during a medication- or placebo-assisted quit attempt. Among smokers with histories of or current mental health conditions, the risk of experiencing a moderate to severe NPSAE was also associated with female sex, younger age, a history of a substance use disorder, and prior treatment with either NRT or bupropion. Importantly, the risk for developing clinically significant NPSAEs was not associated with any treatment or particular category of psychiatric disorder, nor were there interactions among any of the treatments and the independent variables tested in our models.
Comparing our results with related studies obtained via a literature search through August 2017 adds much new information to the field. To our knowledge, this is the first report that identifies easily obtainable patient-level and mental health-related characteristics, with the potential to help predict who is at heightened risk to experience moderate to severe NPSAEs among smokers making a quit attempt. Its uniqueness stems from the fact that EAGLES was designed precisely to evaluate such adverse effects and used both semi-structured diagnostic interviews (e.g., SCID-I and SCID-II) and validated, reliable assessment tools (C-SSRS and HADS) to obtain baseline clinical characteristics that could be used to retrospectively predict NPSAEs. While the literature preceding EAGLES has tracked the occurrence of serious AEs, ${ }^{19-22}$ those more narrowly defined outcomes span multiple organ

Table 4 Regression Results of Screening Assessment of Predictors of NPSAE (NPC)

\begin{tabular}{|c|c|c|c|}
\hline Covariate & $\begin{array}{l}\text { Increase in rate of primary } \\
\text { NPSAE endpoint, \% (SE) }\end{array}$ & $\begin{array}{l}P \text { value for effect } \\
\text { of covariate }\end{array}$ & $\begin{array}{l}P \text { value for treatment- } \\
\text { by-covariate interaction }\end{array}$ \\
\hline Age (1-year increase) & $0.0(0.0)$ & 0.48 & 0.54 \\
\hline Sex (male vs. female) & $0.2(0.4)$ & 0.64 & 0.62 \\
\hline Race* $^{*}$ & & & 0.74 \\
\hline White vs. Black & $3.4(0.8)$ & $<0.001$ & - \\
\hline Other vs. Black & $3.1(1.3)$ & 0.02 & - \\
\hline BMI (1-unit increase) & $-0.0(0.0)$ & 0.52 & 1.00 \\
\hline FTCD (1-unit increase) ${ }^{\dagger}$ & $0.1(0.1)$ & 0.30 & 0.95 \\
\hline Age of starting smoking (1-year increase) & $-0.0(0.0)$ & 0.27 & 0.78 \\
\hline Prior varenicline use (yes vs. no) & $-0.2(0.7)$ & 0.77 & 0.52 \\
\hline Prior bupropion use (yes vs. no) & $2.0(1.1)$ & 0.06 & 0.58 \\
\hline Prior NRT use (yes vs. no) & $1.0(0.6)$ & 0.10 & 0.09 \\
\hline Alcohol/substance use disorder history (yes vs. no) & Too few subjects $(n=8)$ & & \\
\hline HADS anxiety total (1-point increase) & $\begin{array}{l}0.3(0.1) \\
\text { Or } 1.0 \% \text { increase per } 2.9-\text { unit } \\
\text { increase in HADS anxiety total }\end{array}$ & $<0.001$ & 0.09 \\
\hline HADS depression total (1-point increase) & $0.1(0.1)$ & 0.28 & 0.14 \\
\hline Prior $S I / S B(C-S S R S)$ (yes vs. no) & $4.8(1.8)$ & 0.008 & 0.38 \\
\hline Aggression score (BPAQ) (1-unit increase) & $0.0(0.0)$ & 0.87 & 0.26 \\
\hline $\begin{array}{l}\text { Baseline psychotropic medication including } \\
\text { sleeping aids (yes vs. no) }\end{array}$ & $0.5(0.9)$ & 0.58 & 0.09 \\
\hline
\end{tabular}

Abbreviations: BMI body mass index, BPAQ Buss-Perry Aggression Questionnaire, C-SSRS Columbia-Suicide Severity Rating Scale, FTCD Fagerström Test for Cigarette Dependence, HADS Hospital Anxiety and Depression Scale, NPC non-psychiatric cohort, NPSAE neuropsychiatric adverse event, NRT nicotine replacement therapy (transdermal nicotine patch), SB suicidal behavior, SE standard error, SI suicidal ideation

*Only two NPSAEs in Blacks

+Categorization into three groups was not significant, $P=0.68$

A significant effect was found in the screening assessment for covariates in italics 
Table 5 Results of Stepwise Regression Analysis of Predictors of NPSAE (NPC)

\begin{tabular}{lll}
\hline \hline Covariate & $\begin{array}{l}\text { Increase in rate of primary } \\
\text { NPSAE endpoint, \% (SE) }\end{array}$ & $\begin{array}{c}\boldsymbol{P} \text { value for effect } \\
\text { of covariate }\end{array}$ \\
\hline Race & & 0.003 \\
White vs. Black & $2.5(0.8)$ & 0.39 \\
Other vs. Black & $1.3(1.5)$ & 0.02 \\
HADS anxiety total (1-point increase) & $0.3(0.1)$ & Or 1.0\% increase per 4.0-unit \\
& increase in HADS anxiety total score & 0.02 \\
Prior SI/SB (C-SSRS) (yes vs. no) & 4.1 (1.8) & \\
\hline
\end{tabular}

Abbreviations: C-SSRS Columbia-Suicide Severity Rating Scale, HADS Hospital Anxiety and Depression Scale, NPC non-psychiatric cohort, NPSAE neuropsychiatric adverse event, SB suicidal behavior, SE standard error, SI suicidal ideation

systems and lack both the sensitivity and specificity of our NPSAE evaluation battery.

Three characteristics, two of which were related to smokers' mental functioning, were found to have the greatest predictive potential regardless of the individual's psychiatric history. Having a prior history of SI or SB was associated with a $4.4 \%$ increase in the rate of the primary NPSAE endpoint among smokers in the PC, and with a $4.1 \%$ increase among smokers in the NPC after controlling for other factors. While some of this association may be explained by the fact that EAGLES' primary safety endpoint included SI and there was intensive monitoring of such events during the trial with the CSSRS, this finding is important. That smoking and suicidal thinking and behaviors are linked is well established. ${ }^{23-25}$ However, our finding that past SI/SB predicts the likelihood of one experiencing a serious NPS adverse effect during a medication-assisted quit attempt is novel. Moreover, that prior history of SI/SB was independently associated with elevated risk - even after considering other factors such as measures of cigarette addiction itself or of psychiatric illness-related characteristics - speaks to the potential value of obtaining such a history in all smokers who are attempting to quit smoking.

Even subtle elevations in anxiety symptoms at baseline were also found to predict increased occurrence of clinically significant NPSAEs in smokers with and without psychiatric disorders. Thus, for every 3.9-unit increase in HADS anxiety subscale scores among PC smokers, or 4.0-unit increase among NPC participants, the absolute risk of occurrence of the NPSAE endpoint was increased by $1 \%$. The HADS anxiety subscale scoring ranges from 0 to 21 units with each of the seven scale items scored from 0 (no symptom present) to 3 (markedly present). While mean HADS anxiety scores in the EAGLES participants were in the normal range (0-7 units), PC group participants had higher mean HADS scores than NPC smokers. Also, the $\sim 4$-unit elevation observed that signaled increased NPSAE risk may have been linked to some of these smokers having HADS anxiety scores in the 8-10 units range (suggestive of an anxiety state), or $\geq 11$ units, which is the cut-off score marking the probable presence of some type of anxiety disorder. That the increased HADS score predicted adverse effects, while a diagnosis of a frank Axis I anxiety disorder was not associated with increased risk, indicates that a smoker's current anxiety state prior to making a quit attempt may be a more relevant predictor, and that anxiety is a symptom which spans multiple psychiatric and medical disorders. While, to our knowledge, this is the first report of anxiety symptoms being linked to smokers experiencing AEs during a medication-assisted quit attempt, it is noteworthy that patients' anxiety levels have been linked to severity of nicotine withdrawal $^{5,6}$ and to increased likelihood of AEs in patients taking antidepressant ${ }^{26,27}$ or antiepileptic medications. ${ }^{28,}{ }^{29}$ It is also worth noting that anxiety symptoms, and not depressive symptoms also measured on the HADS, were associated with increased risk - an observation also made with antiepileptic drug use. ${ }^{28}$

The demographic and clinical characteristics we found to be associated with clinically significant NPSAEs varied between cohorts, with the exception of White versus Black race, which was found in both cohorts in the screening assessment but was only retained in the stepwise model among smokers in the NPC. There is little information available on how such racial/ ethnic differences affect NPS safety risk, and more research on this topic is warranted. However, to the extent that quitting smoking evokes stress in some individuals, ${ }^{30}$ it is interesting to note that Whites have a more robust hypothalamic-pituitaryadrenal axis response to a psychologic stressor than Blacks, ${ }^{31}$ implying a potential neurobiologic racial difference in stress reactivity. Among smokers with histories of or current mental health conditions, being younger and female was retained in the final regression model after controlling for other variables. Female sex has been linked to milder side effects such as sleep problems and application site reactions in smokers using transdermal nicotine patches, ${ }^{2}$ and both female sex and younger age have been associated with risk for adverse effects from antiepileptic drugs ${ }^{28}$ - the only other class of medications we could find to systematically evaluate such predictors.

Variables associated with cigarette addiction itself and mental health-related factors also predicted additional unique variance in smokers with mental health conditions. Two proxy measures of cigarette dependence severity - prior use of bupropion and previous treatment with NRT-were both retained in the final regression model. The stepwise process eliminated the potentially significant FTCD as a predictor once these other variables were included. We interpret that 
observation cautiously, but we think it likely reflects the more severely dependent smokers being treated more often with NRT and bupropion in the past. Regarding the latter drug, it may be an artifact because our record of prior bupropion use also included use of the medication as an antidepressant. To that end, it is also noteworthy that indicators of psychiatric illness complexity, such as having a comorbid alcohol or substance use disorder, were associated with increased NPSAE occurrence. Taken together then, especially alongside the aforementioned effects of past history of SI/SB and increased state anxiety symptoms, the picture that emerges is that smokers with more complex psychiatric histories and greater severity of nicotine dependence are at greatest risk to develop clinically significant NPSAEs during a medicationassisted quit attempt.

Our study has several limitations that have been documented in detail elsewhere ${ }^{1}$ but bear mentioning again. First, we selected a population of smokers with histories of or currently stable mental health conditions; thus, these findings may not generalize to smokers with unstable or untreated psychiatric disorders. Second, because misuse of alcohol and other drugs causes psychiatric symptoms and mimics psychiatric syndromes, ${ }^{32}$ we excluded smokers with current substance use disorders, further limiting generalizability. Third, the frequent monitoring of this randomized clinical trial might not capture a real-world medication-assisted quit attempt. Fourth, although EAGLES is the largest placebo-controlled smoking cessation pharmacotherapy trial ever conducted, some of the subcohorts in the PC were smaller than others and power to detect effects may be limited. Fifth, EAGLES excluded individuals smoking $<10$ cigarettes per day, so our findings might not generalize to lighter smokers. Sixth, specific to this secondary analysis, there was no comparator involving smokers not attempting to stop. Finally, attrition occurred across all treatment arms and in both cohorts; thus, missing data could have affected the results.

In summary, regardless of the cohort or the medication used, White smokers with past histories of SI and/or SB and current symptoms of anxiety were more likely to experience clinically significant NPSAEs when they tried to quit. Among smokers with psychiatric disorders, younger age, female sex, history of comorbid substance use disorders, and proxy measures of nicotine dependence or psychiatric illness severity also predicted greater NPSAE risk. While to our knowledge this is the first report of patient-level characteristics associated with clinically significant NPSAEs in smokers making a quit attempt, our findings extend those of milder AEs observed with NRT use and adverse effects linked to antidepressant and antiepileptic medication use. Although the incidence of NPSAEs is low in this clinical trial setting and unrelated to any of the study medications, awareness of these pre-existing factors in smokers through the use of already recommended mental health screening procedures in clinical settings ${ }^{33,34}$ is prudent as they might signal heightened NPSAE risk in individuals trying to quit smoking with and without using licensed pharmacotherapies.

Future studies should examine these risk factors in samples of psychiatrically unstable smokers.

Contributors: Editorial support (in the form of creating tables and figures, formatting references, collating review comments, and proofing and formatting for submission) was provided by Anne Jakobsen, MSc, Engage Scientific, Horsham, UK.

Corresponding Author: Robert M. Anthenelli, MD; Department of Psychiatry, University of California, San Diego, La Jolla, CA, USA (e-mail: ranthenelli@ucsd.edu).

Author Contribution Sponsor employees, with input from academic authors, designed the study. The sponsors supported the conduct of the trial, monitored study sites, and collected and analyzed the data; all authors had full access to the data. The lead academic (corresponding) author (RMA) prepared the initial draft of the manuscript and had final responsibility for the decision to submit for publication.

Funders Editorial support was funded by Pfizer. The EAGLES trial was funded by Pfizer and GlaxoSmithKline. EAGLES was a postmarketing requirement in the USA and Europe for Pfizer and GlaxoSmithKline.

\section{Compliance with Ethical Standards:}

Upon request, and subject to certain criteria, conditions and exceptions (see https://www.pfizer.com/science/clinical-trials/trial-dataand-results for more information), Pfizer will provide access to individual de-identified participant data from Pfizer-sponsored global interventional clinical studies conducted for medicines, vaccines and medical devices (1) for indications that have been approved in the USA and/or EU or (2) in programs that have been terminated (i.e., development for all indications has been discontinued). Pfizer will also consider requests for the protocol, data dictionary, and statistical analysis plan. Data may be requested from Pfizer trials 24 months after study completion. The de-identified participant data will be made available to researchers whose proposals meet the research criteria and other conditions, and for which an exception does not apply, via a secure portal. To gain access, data requestors must enter into a data access agreement with Pfizer.

Conflict of Interest: RMA reports his university receiving grants from Alkermes and Pfizer and providing consulting and/or advisory board services to Arena Pharmaceuticals, Cerecor, Pfizer, and US WorldMeds. RMA's writing of this manuscript was supported, in part, by National Institute on Alcohol Abuse and Alcoholism Grant no. U01 AAO13641, and National Institute on Drug Abuse (NIDA) Grant no. UO1 DA041731, and NIDA/Veterans Affairs Cooperative Study no. 1033. NLB reports consulting and/or advisory board services to GlaxoSmithKline, Pfizer, and Achieve Life Sciences and is a paid expert witness in litigation against tobacco companies. RW reports grants from Johnson \& Johnson and Pfizer and personal fees for consulting and/or advisory board services to GlaxoSmithKline, Johnson \& Johnson, and Pfizer. RW's writing of this manuscript was supported, in part, by Cancer Research United Kingdom. AEE reports grants from Forum Pharmaceuticals and $P f i z e r$ and personal fees for advisory board services from Pfizer, Reckitt Benckiser, and Alkermes. AEE's writing of the manuscript was supported by a NIDA Career Award in Patient-Oriented Research, K24 DA030443. MG, TM, CR, DL, and LSA are employees and stockholders of Pfizer. AK is a PAREXEL employee working on behalf of GlaxoSmithKline.

Disclaimer: The opinions expressed in this article are those of the authors and do not necessarily reflect the views of their employers. 
Prior Presentations Parts of this manuscript were presented as a poster at the $23^{\text {rd }}$ Annual Meeting of the Society for Research on Nicotine and Tobacco, March 8-11, 2017, Florence, Italy.

Publisher's Note Springer Nature remains neutral with regard to jurisdictional claims in published maps and institutional affiliations.

\section{REFERENCES}

1. Anthenelli RM, Benowitz NL, West R, St Aubin L, McRae T, Lawrence D, et al. Neuropsychiatric safety and efficacy of varenicline, bupropion, and nicotine patch in smokers with and without psychiatric disorders (EAGLES): a double-blind, randomised, placebo-controlled clinical trial. Lancet. 2016;387(10037):2507-20.

2. Gourlay SG, Forbes A, Marriner T, McNeil JJ. Predictors and timing of adverse experiences during transdermal nicotine therapy. Drug Saf. 1999;20(6):545-55.

3. Leventhal AM, Ramsey SE, Brown RA, LaChance HR, Kahler CW. Dimensions of depressive symptoms and smoking cessation. Nicotine Tob Res. 2008;10(3):507-17.

4. Schnoll RA, Leone FT, Hitsman B. Symptoms of depression and smoking behaviors following treatment with transdermal nicotine patch. J Addict Dis. 2013;32(1):46-52.

5. Morrell HE, Cohen LM, al'Absi M. Physiological and psychological symptoms and predictors in early nicotine withdrawal. Pharmacol Biochem Behav. 2008;89(3):272-8.

6. Farris SG, Zvolensky MJ, Otto MW, Leyro TM. The role of distress intolerance for panic and nicotine withdrawal symptoms during a biological challenge. J Psychopharmacol. 2015;29(7):783-91.

7. Ben Taleb Z, Ward KD, Asfar T, Jaber R, Auf R, Maziak W. Predictors of nicotine withdrawal symptoms: findings from the first randomized smoking cessation trial in a low-income country setting. Int $\mathrm{J}$ Public Health. 2016;61(6):701-8.

8. World Medical Association. WMA Declaration of Helsinki - Ethical Principles for Medical Research Involving Human Subjects. Available at: https://www.wma.net/policies-post/wma-declaration-of-helsinki-ethical-principles-for-medical-research-involving-human-subjects / . Accessed Dec 142018.

9. International Conference on Harmonisation. Guideline For Good Clinica Practice E6(R2). Available at: https://www.ich.org/fileadmin/Public Web_Site/ICH_Products/Guidelines/Efficacy/E6/E6_R2__Step_4_ 2016_1109.pdf. Accessed Dec 142018.

10. First MB, Spitzer RL, Gibbon M, Williams JBW. Structured Clinical Interview for DSM-IV Axis I Disorders (SCID-I). In: Rush Jr AJ, First MB, Blacker D, editors. Handbook of Psychiatric Measures. 2nd ed. Arlington, VA, US: American Psychiatric Publishing Inc.; 2008:40-3.

11. American Psychiatric Association. Diagnostic and Statistical Manual of Mental Disorders, 4th Edition, Text Revison. Available at: https://dsm. psychiatryonline.org/doi/abs/10.1176/appi.books.9780890420249. dsm-iv-tr. Accessed Dec 142018

12. First MB, Gibbon M, Spitzer RL, Williams JBW, Benjamin LS. Structured Clinical Interview for DSM-IV Axis II Personality Disorders (SCID-II). Washington, D.C.: American Psychiatric Press, Inc.; 1997.

13. First MB, Spitzer RL, Gibbon M, Williams JBW. Structured Clinical Interview for DSM-IV-TR Axis I Disorders, Research Version, Patient Edition. (SCID-I/P). New York: Biometrics Research, New York State Psychiatric Institute; November 2002.

14. Fagerström K. Determinants of tobacco use and renaming the FTND to the Fagerstrom Test for Cigarette Dependence. Nicotine Tob Res. 2012;14(1):75-8.

15. Buss AH, Perry M. The aggression questionnaire. J Pers Soc Psychol. 1992;63(3):452-9.
16. Posner K, Brown GK, Stanley B, Brent DA, Yershova KV, Oquendo MA, et al. The Columbia-Suicide Severity Rating Scale: initial validity and internal consistency findings from three multisite studies with adolescents and adults. Am J Psychiatry. 2011;168(12):1266-77.

17. Zigmond AS, Snaith RP. The hospital anxiety and depression scale. Acta Psychiatr Scand. 1983;67(6):361-70.

18. Anthenelli RM, Morris C, Ramey TS, Dubrava SJ, Tsilkos K, Russ C, et al. Effects of varenicline on smoking cessation in adults with stably treated current or past major depression: a randomized trial. Ann Intern Med. 2013;159(6):390-400.

19. Stead LF, Perera R, Bullen C, Mant D, Hartmann-Boyce J, Cahill K, et al. Nicotine replacement therapy for smoking cessation. Cochrane Database Syst Rev. 2012;11:CD000146.

20. Hughes JR, Stead LF, Hartmann-Boyce $\mathbf{J}$, Cahill $\mathbf{K}$, Lancaster $\mathbf{T}$. Antidepressants for smoking cessation. Cochrane Database Syst Rev. 2014; 1:CD000031

21. Cahill K, Stevens S, Perera R, Lancaster T. Pharmacological interventions for smoking cessation: an overview and network meta-analysis. Cochrane Database Syst Rev. 2013;5:CD009329.

22. Cahill K, Lindson-Hawley N, Thomas KH, Fanshawe TR, Lancaster T. Nicotine receptor partial agonists for smoking cessation. Cochrane Database Syst Rev. 2016;5:CD006103.

23. Hughes JR. Smoking and suicide: a brief overview. Drug Alcohol Depend. 2008;98(3): 169-78.

24. Covey LS, Berlin I, Hu MC, Hakes JK. Smoking and suicidal behaviours in a sample of US adults with low mood: a retrospective analysis of longitudinal data. BMJ Open. 2012;2(3):e000876.

25. Evins AE, Korhonen T, Kinnunen TH, Kaprio J. Prospective association between tobacco smoking and death by suicide: a competing risks hazard analysis in a large twin cohort with 35-year follow-up. Psychol Med. 2017;47(12):2143-54.

26. Gaspersz R, Lamers F, Kent JM, Beekman AT, Smit JH, van Hemert AM, et al. Anxious distress predicts subsequent treatment outcome and side effects in depressed patients starting antidepressant treatment. J Psychiatr Res. 2017;84:41-8.

27. Shankman SA, Gorka SM, Katz AC, Klein DN, Markowitz JC, Arnow BA, et al. Side effects to antidepressant treatment in patients with depression and comorbid panic disorder. J Clin Psychiatry. 2017;78(4):433-40.

28. Kim SK, Park SP, Kwon OY. Impact of depression and anxiety on adverse event profiles in Korean people with epilepsy. Epilepsy Behav. 2015;46: 185-91.

29. Perucca P, Jacoby A, Marson AG, Baker GA, Lane S, Benn EK, et al. Adverse antiepileptic drug effects in new-onset seizures: a case-control study. Neurology. 2011;76(3):273-9.

30. Minami H, Frank BE, Bold KW, McCarthy DE. Ecological momentary analysis of the relations among stressful events, affective reactivity, and smoking among smokers with high vs. low depressive symptoms during a quit attempt. Addiction. 2017.

31. Chong RY, Uhart M, McCaul ME, Johnson E, Wand GS. Whites have a more robust hypothalamic-pituitary-adrenal axis response to a psychological stressor than blacks. Psychoneuroendocrinology. 2008;33(2):24654.

32. Shivani R, Goldsmith RJ, Anthenelli RM. Alcoholism and Psychiatric Disorders: Diagnostic Challenges. Available at: https://pubs.niaaa.nih. gov/publications/arh26-2/90-98.htm. Accessed Dec 142018.

33. LeFevre ML, Force USPST. Screening for suicide risk in adolescents, adults, and older adults in primary care: U.S. Preventive Services Task Force recommendation statement. Ann Intern Med. 2014;160(10):71926.

34. Siu AL, Force USPST, Bibbins-Domingo K, Grossman DC, Baumann LC, Davidson KW, et al. Screening for Depression in Adults: US Preventive Services Task Force Recommendation Statement. JAMA. 2016;315(4):380-7. 